June 7,2021

\title{
Evaluation of the BCS Approximation for the Attractive Hubbard Model in One Dimension
}

\author{
F. Marsiglio \\ Neutron $\&$ Condensed Matter Science \\ AECL, Chalk River Laboratories, Chalk River, Ontario, Canada KOJ 1JO, \\ Dept. of Physics \& Astronomy, McMaster University, Hamilton, Ontario L8S $4 M 1$
}

\begin{abstract}
The ground state energy and energy gap to the first excited state are calculated for the attractive Hubbard model in one dimension using both the Bethe Ansatz equations and the variational BCS wavefunction. Comparisons are provided as a function of coupling strength and electron density. While the ground state energies are always in very good agreement, the BCS energy gap is sometimes incorrect by an order of magnitude, particularly at half-filling. Finite size effects are also briefly discussed for cases where an exact solution in the thermodynamic limit is not possible. In general, the BCS result for the energy gap is poor compared to the exact result.
\end{abstract}

\section{INTRODUCTION}

The discovery of high-temperature superconductivity has motivated a considerable effort over the last decade. In particular, many theoretical models have been proposed to explain both the superconductivity and some unusual normal state properties [1]. As far as superconductivity is concerned, model building has proceeded on at least two paths. The first includes "realistic" model Hamiltonians, often two dimensional (since the $\mathrm{CuO}_{2}$ planes have been deemed to be the essential structure for electron conduction). The price of such realism 
is that exact solutions are impossible, and a mean field or BCS [2] solution is required. The second path attempts to remedy this deficiency by seeking exact solutions to a restricted class of models, often in one (and more recently in infinite) dimension. These models are often unrealistic, and are sometimes exactly solvable only in restricted parameter regimes. Attempts to bridge the gap between these two modes of theoretical work are becoming more commonplace in recent years. Two examples are spin fluctuation theories, where Fluctuation Exchange calculations are often compared to Monte Carlo calculations for the Hubbard model [3], and Hubbard-like models that include occupation-dependent hopping rates [4]. In this latter example BCS calculations have been compared with exact diagonalization studies on finite systems.

In these and other examples, comparison of BCS or some other approximation scheme with exact results has always been hampered by some deficiency, often finite size effects (one exception is mentioned below). Yet an exact solution to the attractive Hubbard model in one dimension has been available for almost thirty years, via Bethe Ansatz techniques [5].

In this paper a thorough investigation of the attractive Hubbard model will be presented, using both Bethe Ansatz techniques and BCS solutions. The study is necessarily confined to one dimension. It is not yet clear to what extent conclusions obtained in one dimension carry over to higher dimensions. Nonetheless, we were encouraged by the results of Quick et al. [6], who performed a study similar to the present one using a model of a one-dimensional electron-gas with pairwise-attractive delta-function interactions, first solved exactly by Gaudin [7] and Yang [8]. This is a one parameter model with a dimensionless coupling constant inversely proportional to the electron density. Quick et al. [6] found that BCS theory gave an accurate estimate of the ground state energy over all ranges of the coupling constant. Quantum fluctuations are most severe in one dimension, so the agreement should improve as the dimension is increased.

Part of the purpose of the present study is to test the accuracy of BCS as "an interpolation scheme" [9] [0] over all coupling strengths. As already noted in Ref. [9], BCS theory is exact in the weak and strong coupling limits. The present model has two parameters, electron 
density as well as coupling strength, and the question of how well the interpolation scheme works as a function of electron density is addressed. Moreover, the gap to the first excited state will also be calculated, as this quantity provides a much more revealing test of the BCS approximation than does the ground state energy.

We begin by outlining the model and the theoretical treatments in the following section. For the exact solution we rely on previous work by Lieb and $\mathrm{Wu}$ [5] and by Bahder and Woynarovich [11]. We mention some trivial but important points regarding the solution of the resulting equations, for both the ground state energy, and the excitation gap. For completeness we also outline the BCS solution, which is straightforward. Results are presented in the following section, followed by a summary.

\section{FORMALISM}

\section{A. The Model}

The one dimensional Hubbard Hamiltonian is given by

$$
H=-t \sum_{i, \sigma}\left(c_{i \sigma}^{\dagger} c_{i+1, \sigma}+c_{i+1, \sigma}^{\dagger} c_{i \sigma} \quad\right)+U \sum_{i} n_{i \uparrow} n_{i \downarrow}
$$

where the operator $c_{i \sigma}^{\dagger}\left(c_{i \sigma}\right)$ creates (annihilates) an electron with spin $\sigma$ at site $i$ and $n_{i \sigma}$ is the electron number operator with spin $\sigma$ at site $i$. The parameter $t$ is the hopping rate for electrons and merely sets the overall energy scale. The parameter $U$ is negative in the attractive case, so that $|U| / t$ is a dimensionless measure of the coupling strength. The second parameter is the electron density, $n \equiv \frac{1}{N} \sum_{i, \sigma}<n_{i \sigma}>$, where $N$ is the number of lattice sites, and the expectation value is taken with respect to the ground state.

This model has particle-hole symmetry, so we will concern ourselves with electron densities less than half-filling. In addition, symmetries exist between the repulsive and attractive Hubbard models, such that [5]

$$
E\left(M, M^{\prime} ;-|U|\right)=-M|U|+E\left(M, N-M^{\prime} ;|U|\right)
$$

where $E\left(M, M^{\prime} ;|U|\right)$ is the ground state energy of $H$ (with $U=|U|$ ) with $M$ down-spins 
and $M^{\prime}$ up-spins $\left(M+M^{\prime}=N_{e}\right.$, the total number of electrons). The total z-component of electron spin, $S_{z}$, is given by $S_{z} \equiv \frac{1}{2}\left(M^{\prime}-M\right)$. For the ground state, $S_{z}=0$.

\section{B. The Lieb-Wu Equations: Finite Systems}

An exact solution to the Hubbard model was first provided by Lieb and $\mathrm{Wu}$ [5], using Bethe Ansatz techniques. A particularly enlightening derivation is provided by Sutherland in Ref. [12]. The resulting equations to determine the wavevectors of the $N_{e}$ electrons are:

$$
k_{j}=\frac{2 \pi}{N} I_{j}+\frac{1}{N} \sum_{\beta=1}^{M} \theta\left(2 \sin k_{j}-2 \Lambda_{\beta}\right), \quad j=1,2,3, \ldots, N_{e} .
$$

An auxiliary set of $M$ real numbers, the $\Lambda_{\alpha}$ 's are determined through the set of equations

$$
\sum_{j=1}^{N_{e}} \theta\left(2 \sin k_{j}-2 \Lambda_{\alpha}\right)=2 \pi J_{\alpha}-\sum_{\beta=1}^{M} \theta\left(\Lambda_{\alpha}-\Lambda_{\beta}\right), \quad \alpha=1,2,3, \ldots, M,
$$

where

$$
\theta(p) \equiv-2 \tan ^{-1}(2 p t / U),-\pi \leq \theta<\pi
$$

Here the $I_{j}$ are consecutive integers (half-odd integers) if $M$ is even (odd), and the $J_{\alpha}$ are consecutive integers (half-odd integers) if $M^{\prime}$ is odd (even). For the ground state we choose them to be (separately) clustered around zero (both negative and positive). In general, for finite size systems, their $\operatorname{sum}\left(\frac{1}{N}\left(\sum_{j} I_{j}+\sum_{\alpha} J_{\alpha}\right)\right)$ does not give zero, so that the total momentum of the ground state in some sectors is not zero. For the attractive Hubbard model $M$ and $M^{\prime}$ are kept close to one another so that the maximum number of electrons with opposite spin can pair to take advantage of the attractive $|U|$.

These equations can be iterated to convergence using a standard Newton-Raphson algorithm [13], although some damping of solutions and judicious starting points are sometimes required. Once the $k_{j}$ have been obtained, the ground state energy is given by

$$
E=-2 t \sum_{j=1}^{N_{e}} \cos k_{j}
$$

One way to determine the energy of the first excited state is to compare the energy of two isolated systems with $N_{e}$ electrons in one and $N_{e}+2$ in the other, with two isolated systems with $N_{e}+1$ in each. This yields the pair binding energy, 


$$
\epsilon_{b}\left(N_{e}\right)=2 E\left(N_{e}+1\right)-E\left(N_{e}\right)-E\left(N_{e}+2\right) .
$$

Here we have used a single argument for the energy to indicate the total number of electrons — it is understood that $M$ and $M^{\prime}$ are either equal (if $N_{E}$ is even) or differ by one (if $N_{e}$ is odd). The single particle gap, $\Delta\left(N_{e}\right)$, is then given by

$$
\Delta\left(N_{e}\right)=\epsilon\left(N_{e}\right) / 2 .
$$

In this model the gap value is positive for $N_{e}$ even and negative for $N_{e}$ odd, so that the overall curvature is in fact non-negative (see the second reference in [⿶凵⿴囗十). Thus, to calculate the gap value in this manner requires a distinction between even and odd numbers of electrons, a distinction which is unfortunately lost in the thermodynamic limit. Nonetheless, finite size effects can be monitored by increasing the system size until convergence is achieved. This becomes quite time-consuming, however, for weak coupling (where large system sizes are required for convergence), so that an alternative method is required, as discussed next.

\section{The Lieb-Wu Equations: Bulk Limit}

As $N \rightarrow \infty, k$ and $\Lambda$ become distributed throughout the first Brillouin zone and the real axis, respectively, with density functions $\rho(k)$ and $\sigma(\Lambda)$, respectively 12. The above equations become the Lieb-Wu integral equations:

$$
\rho(k)=\frac{1}{2 \pi}+\frac{\cos k}{\pi} \int_{-B}^{B} \frac{|U| / 4 t}{(U / 4 t)^{2}+(\lambda-\sin k)^{2}} \sigma(\lambda) d \lambda,
$$

with $\sigma(\lambda)$ determined self-consistently from

$$
\frac{1}{\pi} \int_{-Q}^{Q} \frac{|U| / 4 t}{(U / 4 t)^{2}+(\lambda-\sin k)^{2}} \rho(k) d k=\sigma(\lambda)+\frac{1}{\pi} \int_{-B}^{B} \frac{|U| / 2 t}{(U / 2 t)^{2}+\left(\lambda-\lambda^{\prime}\right)^{2}} \sigma\left(\lambda^{\prime}\right) d \lambda^{\prime},
$$

where $Q$ and $B$ are determined by the following sum rules:

$$
\begin{aligned}
& \int_{-Q}^{Q} \rho(k) d k=1-2 s \\
& \int_{-B}^{B} \sigma(\lambda) d \lambda=\frac{n}{2}-s
\end{aligned}
$$


with $n \equiv N_{e} / N$ and $s \equiv S_{z} / N$. Finally the energy per lattice site is given by

$$
\frac{E}{N}=-|U|\left(\frac{n}{2}-s\right)-2 t \int_{-Q}^{Q} \cos k \rho(k) d k
$$

Note that we have already utilized Eq. (2) in these equations; hence they apply to an attractive Hubbard model with electron density $n$ and magnetization $s$. The ground state is given by $s=0$, so that $Q=\pi$, i.e. the entire Brillouin zone is occupied. These equations converge more easily than do their discrete counterparts given above, i.e. simple iteration is sufficient, and Newton-Raphson (and therefore the inversion of a large matrix) is not required.

However, in principle, information concerning the gap is lost, as the distinction between even and odd numbers of electrons is no longer possible in the bulk limit. Nonetheless, Bahder and Woynarovich [11] use the trick of applying an external magnetic field. Minimization of the energy then determines the magnetization as a function of applied field. The magnetization is zero below a critical value of the field. The energy associated with this critical field gives the gap in the spin excitation spectrum. It turns out that half this energy corresponds exactly with the gap as defined by Eqs. (7) and (8). This is not a priori necessary, as the spin-gap is defined by flipping a spin with fixed electron number, whereas the previous gap was defined by changing the number of electrons in the system.

To determine the gap in the bulk limit, we use a different procedure, which initially follows the method outlined in Ref. [11] (see their section IV where they carry out an analytical calculation in the low and high density limits). As already mentioned, the spingap can be defined as the energy required to flip one spin. Therefore we are interested in the energy difference between the $s=0$ state and the $s=1 / N$ state where $N$ is taken to approach $\infty$. Since $Q=\pi$ for $s=0$, we have from Eq. (11) that $Q=\pi-1 /[N \rho(\pi)]$, using the even symmetry of $\rho(k)$. Substitution into Eq. (10) yields one term on the left-hand side which is $O(1 / N)$. Furthermore, $\rho(k)$ can now be eliminated by substituting Eq. (9) into the remaining integral from $-\pi$ to $\pi$ [1]]: 
$\frac{1}{2 \pi^{2}} \int_{-\pi}^{\pi} \frac{|U| / 4 t}{(U / 4 t)^{2}+(\lambda-\sin k)^{2}}-\frac{2}{\pi N} \frac{|U| / 4 t}{(U / 4 t)^{2}+\lambda^{2}}=\sigma(\lambda)+\frac{1}{\pi} \int_{-B}^{B} d \lambda^{\prime} \sigma\left(\lambda^{\prime}\right) \frac{|U| / 2 t}{(U / 2 t)^{2}+\left(\lambda-\lambda^{\prime}\right)^{2}}$

To first order in $1 / N$ there is no change in the density function $\rho(k)$; however, the same is not true for $\sigma(\lambda)$. To see this we substitute $s=1 / N$ into Eq. (12), and allow $B$ to adjust $\left(B \rightarrow B_{\circ}+\delta B\right)$ to the flipped spin (here the subscript o signifies the solution for $\left.s=0\right)$. The important point is that the electron density $n$ remains constant. Then

$$
\delta B=\frac{1}{2 \sigma\left(B_{\circ}\right)}\left\{\int_{-B_{\circ}}^{B_{\circ}} \sigma(\lambda) d \lambda-\frac{n}{2}+\frac{1}{N}\right\}
$$

where again we have utilized the even symmetry of $\sigma(\lambda)$. The two first terms within the braces do not cancel because there are $O(1 / N)$ corrections to the density function $\sigma(\lambda)$. This is most clearly seen at half-filling, where $\delta B=0$ and the sum rule has apparently changed by $O(1 / N)$. Away from half-filling $\delta B$ is $O(1 / N)$. Substitution of Eq. (15) into Eq. (14) results in

$$
\begin{aligned}
\sigma(\lambda)= & \frac{1}{2 \pi^{2}} \int_{-\pi}^{\pi} d k \frac{|U| / 4 t}{(U / 4 t)^{2}+(\lambda-\sin k)^{2}}-\frac{1}{\pi} \int_{-B_{\circ}}^{B_{\circ}} d \lambda^{\prime} \sigma\left(\lambda^{\prime}\right) \frac{|U| / 2 t}{(U / 2 t)^{2}+\left(\lambda-\lambda^{\prime}\right)^{2}} \\
& -\frac{2}{\pi N} \frac{|U| / 4 t}{(U / 4 t)^{2}+\lambda^{2}}+\frac{\delta B}{\pi} \sigma\left(B_{\circ}\right)\left\{\frac{|U| / 2 t}{(U / 2 t)^{2}+\left(\lambda-B_{\circ}\right)^{2}}+\frac{|U| / 2 t}{(U / 2 t)^{2}+\left(\lambda+B_{\circ}\right)^{2}}\right\}
\end{aligned}
$$

where every term except the first on the right-hand-side contains (explicit or implicit) terms with $O(1 / N)$. This equation is iterated to convergence (with high precision) with some choice for $N$ (eg. $N=10^{4}$ or $10^{5}$ ). The equation for the energy, Eq. (13) becomes

$$
\begin{aligned}
\frac{E}{N}= & -|U| \frac{n}{2}-\frac{2 t}{\pi} \int_{-\pi}^{\pi} d k \cos ^{2} k \int_{-B_{\circ}}^{B_{\circ}} d \lambda \sigma(\lambda) \frac{|U| / 4 t}{(U / 4 t)^{2}+(\lambda-\sin k)^{2}} \\
& +\frac{|U|-4 t}{N}-\delta B \sigma\left(B_{\circ}\right)\left\{\frac{|U| / 4}{(U / 4 t)^{2}+\left(B_{\circ}-\sin k\right)^{2}}+\frac{|U| / 4}{(U / 4 t)^{2}+\left(B_{\circ}+\sin k\right)^{2}}\right\} .
\end{aligned}
$$

The gap energy is then identified as the coefficient of the $1 / N$ term in the energy,

$$
\frac{E}{N} \equiv \frac{E_{\circ}}{N}+\frac{2 \Delta}{N}
$$

The first term on the right-hand-side is obtained by solving the integral equations with $N \rightarrow \infty$, i.e. Eqs. (9-13). Then the left-hand-side is obtained by solving Eqs. (15-17) for $N=10^{4}, 10^{5}$, etc., until the gap extracted from Eq. (18) has converged. 


\section{The BCS Equations}

The derivation of the BCS equations is given in many places [14 and will not be presented here. They are:

$$
1=\frac{|U|}{N} \sum_{k} \frac{1}{2 E_{k}}
$$

with

$$
E_{k}=\sqrt{\left(\epsilon_{k}-\mu-|U| n / 2\right)^{2}+\Delta_{\mathrm{BCS}}^{2}}
$$

and $\epsilon_{k}=-2 t \cos k$, where the sums are carried out over the first Brillouin zone $(-\pi<k \leq \pi)$. We use a finite lattice for simplicity, large enough for the gap to have converged to its bulk limit. An auxiliary equation to determine the electron number density is also required,

$$
n=1-\frac{1}{N} \sum_{k} \frac{\epsilon_{k}-\mu-|U| n / 2}{E_{k}} .
$$

For a given $|U|$ and $n$ these equations are iterated to convergence to determine $\mu$ and $\Delta_{\mathrm{BCS}}$. If the chemical potential lies within the band, then the gap to the first excited state, $\Delta_{\circ}$, is given by $\Delta_{\mathrm{BCS}}$. Otherwise, the gap is defined by the quasiparticle energy at the bottom of the band, i.e.

$$
\begin{aligned}
\Delta_{\circ} & =\Delta_{\mathrm{BCS}} & & \text { for } \mu+|U| n / 2>-2 t \\
& =\sqrt{(2 t+\mu+|U| n / 2)^{2}+\Delta_{\mathrm{BCS}}^{2}} & & \text { for } \mu+|U| n / 2<-2 t
\end{aligned}
$$

$\Delta_{\circ}$ is to be directly compared to the gap discussed in the previous sections. Finally, the total energy is given by

$$
\frac{E_{\mathrm{BCS}}}{N}=\frac{1}{N} \sum_{k} \epsilon_{k}\left(1-\frac{\epsilon_{k}-\mu-|U| n / 2}{E_{k}}\right)-|U|\left(\frac{n}{2}\right)^{2}-\frac{\Delta_{\mathrm{BCS}}^{2}}{|U|} .
$$

Note that the Hartree term is not required in the BCS gap equations (and is often omitted), but it provides an important contribution to the total energy.

\section{RESULTS}




\section{A. The Ground State Energy}

In the dilute limit the BCS solution of the gap equations is exact for all coupling strengths [9]. Of course the BCS approximation also reduces to the non-interacting result in the limit of weak coupling, for any electron density. Finally, BCS theory also becomes exact in the limit of strong coupling, as was appreciated for both the electron gas [15] and the lattice gas [9].

In Fig. 1 the ground state energy is shown as a function of electron density for various values of $U$. We have shown some results for repulsive $U$ [16] along with the analytical results

for $U=0\left(E / N=-\frac{4}{\pi} \sin \frac{n \pi}{2}\right)$ and $U=\infty\left(E / N=-\frac{2}{\pi} \sin n \pi\right)$. Note that agreement is best in weak coupling and in strong coupling, particularly for low electron density. Nonetheless, the BCS energy follows the exact result much more closely in the intermediate regime for this model than appears to be the case for the electron gas model [6]. In fact a strong coupling expansion shows that the ground state energy has corrections of order $(t / U)^{2}$ in both the exact and BCS solutions, whereas for the gas model the BCS solution contained a term linear in the inverse coupling constant which was not present in the exact solution [6].

In Fig. 2 we show the ground state energy as a function of coupling strength, $|U|$ for half-filling $(n=1)$ and quarter-filling $(n=0.5)$. Here it is clear that the BCS theory is most inaccurate for intermediate coupling strength, i.e. $|U| \approx$ bandwidth. Nonetheless the maximum deviation is rather small, $\approx 4 \%$, which occurs at half-filling near $|U|=4 t$. Thus, on the basis of ground state energy calculations, BCS theory appears to be a very accurate theory, even in one dimension. However, in the next subsection we show that the BCS result for the energy gap is far less accurate.

\section{B. The Energy Gap}

The calculation of the energy gap is a much more sensitive test of the accuracy of BCS theory. In Fig. 3 we show the gap as a function of electron density for various values of the interaction strength, $|U|$. As already stated, the BCS gap is exact in the low density limit, regardless of the coupling strength. However, as the electron density increases, the true 
gap decreases for all coupling strengths, while the BCS gap $\left(\Delta_{\circ}\right)$ from Eq. (22) increases for all coupling strengths. The true gap decreases monotonically as a function of electron density, whereas the BCS gap is in general non-monotonic, exhibiting a maximum at some intermediate electron density, which is a function of coupling strength. For sufficiently strong coupling, the BCS gap is monotonically increasing, so that the maximum occurs at half-filling. The most serious errors occur near half-filling, particularly in the weak coupling limit, where BCS theory vastly overestimates the value of the gap.

At half-filling certain analytical results can be obtained. For the true gap we obtain

$$
\Delta(n=1)=\frac{|U|}{2}-2 t+4 t \int_{0}^{\infty} \frac{d \omega}{\omega} \frac{J_{1}(\omega)}{1+e^{\omega|U| / 2 t}}
$$

where $J_{1}(\omega)$ is the Bessel function of the first kind of order 1. This result can be written in a more useful form [18],

$$
\Delta(n=1)=\frac{8 t}{\pi} \sum_{m=1}^{\infty} \frac{1}{2 m-1} K_{1}\left(\frac{2 \pi t}{|U|}(2 m-1)\right),
$$

where $K_{1}(x)$ is the first order modified Bessel function. In weak coupling this reduces to an exponential contribution:

$$
\Delta_{\text {weak }}=\frac{4}{\pi} \sqrt{|U| t} \exp \left\{\frac{-2 \pi t}{|U|}\right\}
$$

In strong coupling the first two terms of Eq. (24) dominate.

The BCS integral can also be performed analytically: we obtain, at half-filling,

$$
\frac{2 \pi t}{|U|}=\frac{1}{\sqrt{1+\delta^{2}}} K\left(\frac{1}{1+\delta^{2}}\right)
$$

where $\delta \equiv \frac{\Delta_{0}}{4 t}$ and $K(k) \equiv \int_{0}^{\pi / 2} \frac{1}{\sqrt{1-k \sin ^{2} \theta}} d \theta$ is the complete elliptic integral of the first kind. Note that $\Delta_{\circ}=\Delta_{\mathrm{BCS}}$ at half-filling. In weak coupling we obtain

$$
\Delta_{\circ \text { (weak) }}=8 t \exp \left(-\frac{2 \pi t}{|U|}\right)
$$

while in strong coupling we have

$$
\Delta_{\circ(\text { strong })}=\frac{|U|}{2}-\frac{2 t^{2}}{|U|}
$$


Thus, the weak coupling BCS gap at half-filling has the familiar activated form, albeit with incorrect prefactors. In strong coupling the first order correction (of order unity) is absent in the BCS solution.

Finally, at zero filling, the exact solution is known analytically [17]:

$$
\Delta=-2 t+\sqrt{\left(\frac{|U|}{2}\right)^{2}+(2 t)^{2}}
$$

and the BCS result is identical. Note that in weak coupling the dependence on $|U|$ is quadratic.

In Fig. 4 we show the gap as a function of coupling strength for zero-, quarter-, and half-filling. As already stated, for zero filling BCS is exact, while for any non-zero filling, the deviations are as shown. BCS theory overestimates the gap by a considerable degree over the entire coupling range shown. Note that the variation of the exact gap with electron density is not significant beyond $|U| / t \approx 2$, and, as remarked earlier, monotonically decreases with increasing electron density. The dependence of the BCS gap on the electron density is not monotonic, and beyond $|U| / t \approx 3$ the ordering is incorrect. In Fig. $4 \mathrm{~b}$ we show the weak coupling regime in greater detail. In this regime the ordering with electron density is correct, but BCS theory greatly overestimates the gap value, as shown.

\section{Finite Size Effects}

The attractive Hubbard model can be solved in the thermodynamic limit, and therefore, for this study, finite size effects do not concern us. However, most models require some sort of numerical solution, often on small system sizes, and are therefore subject to finite size effects. It is therefore of interest to examine how these effects influence the results in both the exact and BCS solution in the present model. To some extent the following conclusions can be applied to other models where an exact solution in the bulk limit is not possible.

To obtain the exact gap it is necessary to solve Eqs.(3-8) for some fixed lattice size, $N$. The BCS gap is obtained from Eqs.(19),22), again for a fixed lattice size, $N$. However, these equations utilize a grand canonical ensemble, so that particle number is fixed only in 
an average sense, through the chemical potential. Thus, all electron densities are possible (except for certain densities where discontinuities may arise); this raises the issue of how applicable the use of the grand canonical ensemble is for small clusters. We partially address this issue here by using the canonical ensemble for clusters with one, two, three and four electrons. This has already been done for a tetrahedral cluster [19], where, for symmetry reasons, the BCS solution is exact for all pair-fillings on a four-site cluster. We use the BCS wavefunctions

$$
\begin{aligned}
& \left|\Psi_{2}>=\sum_{k} g_{k} c_{k \uparrow}^{\dagger} c_{-k_{\downarrow}}^{\dagger}\right| 0> \\
& \left|\Psi_{4}>=\sum_{\substack{k_{1} \\
k_{2}>k_{1}}} C\left(k_{1}, k_{2}\right) c_{k_{1} \uparrow}^{\dagger} c_{-k_{1} \downarrow}^{\dagger} c_{k_{2} \uparrow}^{\dagger} c_{-k_{2} \downarrow}^{\dagger}\right| 0>
\end{aligned}
$$

for two and four electrons, respectively. On a chain of four sites these would correspond to quarter- and half-filling. To accomodate odd numbers of electrons we simply add another electron, whose momentum then determines the total momentum of the ground state wavefunction. For example, for three electrons,

$$
\left|\Psi_{3}>_{q}=\sum_{k \neq q} g_{k}^{\prime} c_{k \uparrow}^{\dagger} c_{-k \downarrow}^{\dagger} c_{q \uparrow}^{\dagger}\right| 0>
$$

and the third electron has the important role of blocking the pair from adopting the $(q,-q)$ pair state. Minimization with respect to the relevant parameters yields equations for the $g_{k}$ or $C\left(k_{1}, k_{2}\right)$ from which the minimum energy can be obtained. The gap energy can then be obtained following the method outlined in Section 2.B. In contrast to the grand canonical method for finite systems, this method generates gap energies for values of the electron density that are commensurate with the lattice size.

In Fig. 5 results for the gap are illustrated as a function of density for $|U|=-2 t$, using all the various methods discussed thus far. The open symbols represent exact solutions for various lattice sizes as indicated in the figure caption. The gap generally decreases as the lattice approaches the bulk limit. The same trend is observed in the BCS solutions, indicated by the various curves. Finally, for $N=4$, the filled circle indicates the result for quarter filling from the canonical BCS equations. 
We have found quite generally that the use of the canonical ensemble always improves the ground state energy, compared to that obtained from the grand canonical ensemble. This is particularly true for electron densities that correspond to an odd number of electrons, and for low electron densities. In fact the conventional BCS theory is variational with respect to $H-\mu N$, and not the Hamiltonian alone, so that for small system sizes this theory gives lower energies than the true ground state energies at low electron densities. This does not violate the variational principle since the BCS wavefunction used spans all particle numbers, and BCS theory never gives a lower energy than the true ground state energy for any particle number. In any event, this distasteful feature is remedied by the use of the canonical ensemble. Of course, as the bulk limit is approached these discrepancies disappear.

However, the use of the canonical ensemble does not universally improve the BCS gap energy for small systems. The case shown in Fig. 5 shows some improvement. For weaker coupling the improvement is insignificant, while for stronger coupling the result from the canonical solution is poorer than the grand canonical one.

It should be clear that finite size effects are significant only in the intermediate to weak coupling regime (the two site system reproduces the correct bulk limit in the strong coupling limit). For the intermediate coupling case shown, the finite size results could mislead one to believe that the gap energy increases as electron density increases from zero. This behaviour is displayed by the BCS results as well, and remains a feature of the BCS solution in the bulk limit (solid line). However, the true energy gap is a monotonically decreasing function of electron density. Furthermore, the gap always decreases as the bulk limit is approached, so that the relative error obtained from a finite system study will always underestimate the true error in the bulk limit. The error in the BCS gap at half-filling, for example, is $20 \%$ for a four site chain, while, in the bulk limit it is $300 \%$. This underestimate worsens for weaker coupling.

Finally, we should note that the finite size effects on the ground state energy are not nearly as severe as just discussed for the energy gap. For example, the ground state energy 
at half-filling for $N=8$ differs from the bulk limit by about $1 \%$ while the gap value is almost a factor of three too large.

\section{SUMMARY}

We have carried out an evaluation of BCS theory for the attractive Hubbard model in one dimension. This is certainly the simplest Hamiltonian with many-body interactions for which an exact solution exists, so that an evaluation in the bulk limit is possible. We hope that the results of this paper can be used for more complicated Hamiltonians where exact solutions are not possible.

The ground state energy is very accurately reproduced by the BCS wavefunction, particularly at low electron densities, and for all coupling strengths. In fact the ground state energy is least accurate at intermediate coupling strengths, at half-filling, and here the error is only a few per cent. This result is encouraging, and ought to improve with increasing dimensionality.

The energy gap is not so accurately reproduced within BCS theory. There is exact agreement in the dilute limit; however the exact result monotonically decreases with electron density while the BCS result increases, at least initially. There is also agreement in the strong coupling limit, and BCS theory displays the same activated form in the weak coupling limit, but with an incorrect prefactor. At intermediate coupling strength the BCS gap overestimates the true gap typically by a factor of two or more. The relative error decreases

(increases) with stronger (weaker) coupling. Over most of the coupling strength regime the maximum error occurs at half-filling.

Finally, we have taken advantage of this exactly solvable case to examine finite size effects. Insofar as these effects occur in other more complicated models, they can be used as cautionary guidelines in future finite system studies.

The relevance to higher dimensionality remains somewhat of an open question. While the accuracy of the BCS ground state energy is most encouraging, it is also clear from this study that this agreement is not a good indicator of the accuracy of the BCS energy gap. 
On the other hand the poor accuracy of the BCS gap in one dimension may well be rectified by proceeding to higher dimension. It is hoped that future investigations of this issue can utilize this study as a benchmark.

\section{Acknowledgements}

I thank Takashi Nakatsukasa for helpful discussions and Graham Lee-Whiting for his derivation of Eq. (25). Partial support from the Natural Science and Engineering Research Council (NSERC) of Canada is acknowledged. 


\section{REFERENCES}

[1] See for example, Proceedings of the International Conference on Materials and Mechanisms of Superconductivity: High Temperature Superconductors IV, edited by P. Wyder (North-Holland, The Netherlands, 1994) Physica C 235-240 1-3502 (1994). This and the previous three conference proceedings (Materials and Mechanisms of Superconductivity: High Temperature Superconductors III, edited by M. Tachiki, Y. Muto and Y. Syono (North-Holland, The Netherlands, 1991) Physica C 185-189 1-2768 (1991); Materials and Mechanisms of Superconductivity: High Temperature Superconductors II, edited by R.N Shelton, W.A. Harrison and N.E. Phillips, (North-Holland, The Netherlands, 1989) Physica C 162-164 1-1747 (1989); High Temperature Superconductors and Materials and Mechanisms of Superconductivity, edited by J. Müller and J.L. Olsen (North-Holland, The Netherlands, 1988) Physica C 153-155 1-1801 (1988)) provide a catalog of the developments since the discovery by Bednorz and Müller.

[2] J. Bardeen, L.N. Cooper and J.R. Schrieffer, Phys. Rev. 108, 1175 (1957)

[3] For references, see a recent review by D.J. Scalapino, Phys. Rep. 250, 329 (1995).

[4] M. Airoldi and A. Parola, Phys. Rev. B 51, 16327 (1995);

H.Q. Lin and J.E. Hirsch, Phys. Rev. B 52, 16155 (1995);

W. Minor (unpublished).

[5] E.H. Lieb and F.Y. Wu, Phys. Rev. Lett. 20, 1445 (1968).

[6] R.M. Quick, C. Esebbag and M. de Llano, Phys. Rev. B 47, 11512 (1993).

[7] M. Gaudin, Phys. Lett. 24A, 55 (1967).

[8] C.N. Yang, Phys. Rev. Lett. 19, 1312 (1967).

[9] P. Nozières and S. Schmitt-Rink, J. Low Temp. Phys. 59, 195 (1985).

[10] A.J. Leggett, J. de Physique, C7, 41, 19 (1980); 
A.J. Leggett, in Modern Trends in the Theory of Condensed Matter, edited by S. Pekalski and J. Przystawa (Springer, Berlin, 1980)p. 13.

[11] T.B. Bahder and F. Woynarovich, Phys. Rev. B 33, 2114 (1986).

[12] B. Sutherland, in Exactly Solvable Problems in Condensed Matter and Relativistic Field Theory, edited by B.S. Shastry, S.S. Jha and V. Singh (Springer-Verlag, Berlin, 1985)p. 1.

[13] Numerical Recipes: The Art of Scientific Computing, by W.H. Press, B.P. Flannery, S.A. Teukolsky and W.T. Vetterling, (Cambridge University Press, Cambridge, 1986)p. 269.

[14] J.R. Schrieffer, Theory of Superconductivity (Benjamin/Cummings, Don Mills, 1964).

[15] D.M. Eagles, Phys. Rev. 186, 456 (1969).

[16] H. Shiba, Phys. Rev. B6, 930 (1972).

[17] J.E. Hirsch, Physica C 179, 317 (1991).

[18] I am indebted to Graham Lee-Whiting for the derivation of this result.

[19] L.M. Falicov and C.R. Proetto, Phys. Rev. B 47, 14407 (1993). 


\section{FIGURES}

Fig. 1 Marsiglio

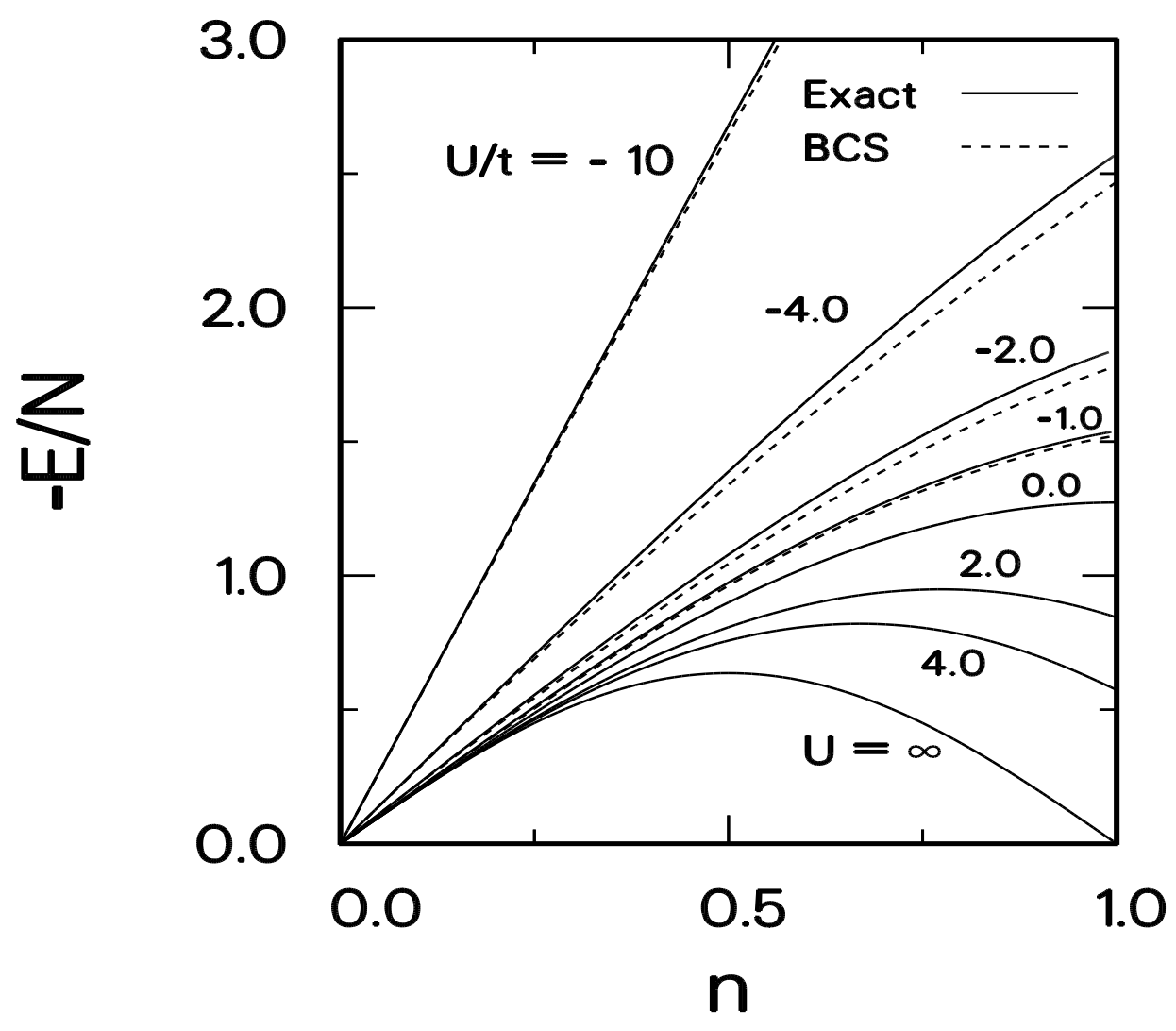

FIG. 1. The ground state energy as a function of electron density for various values of the coupling strength, both repulsive $(U>0)$ and attractive $(U<0)$. BCS results are also shown for comparison. 
Fig. 2 Marsiglio

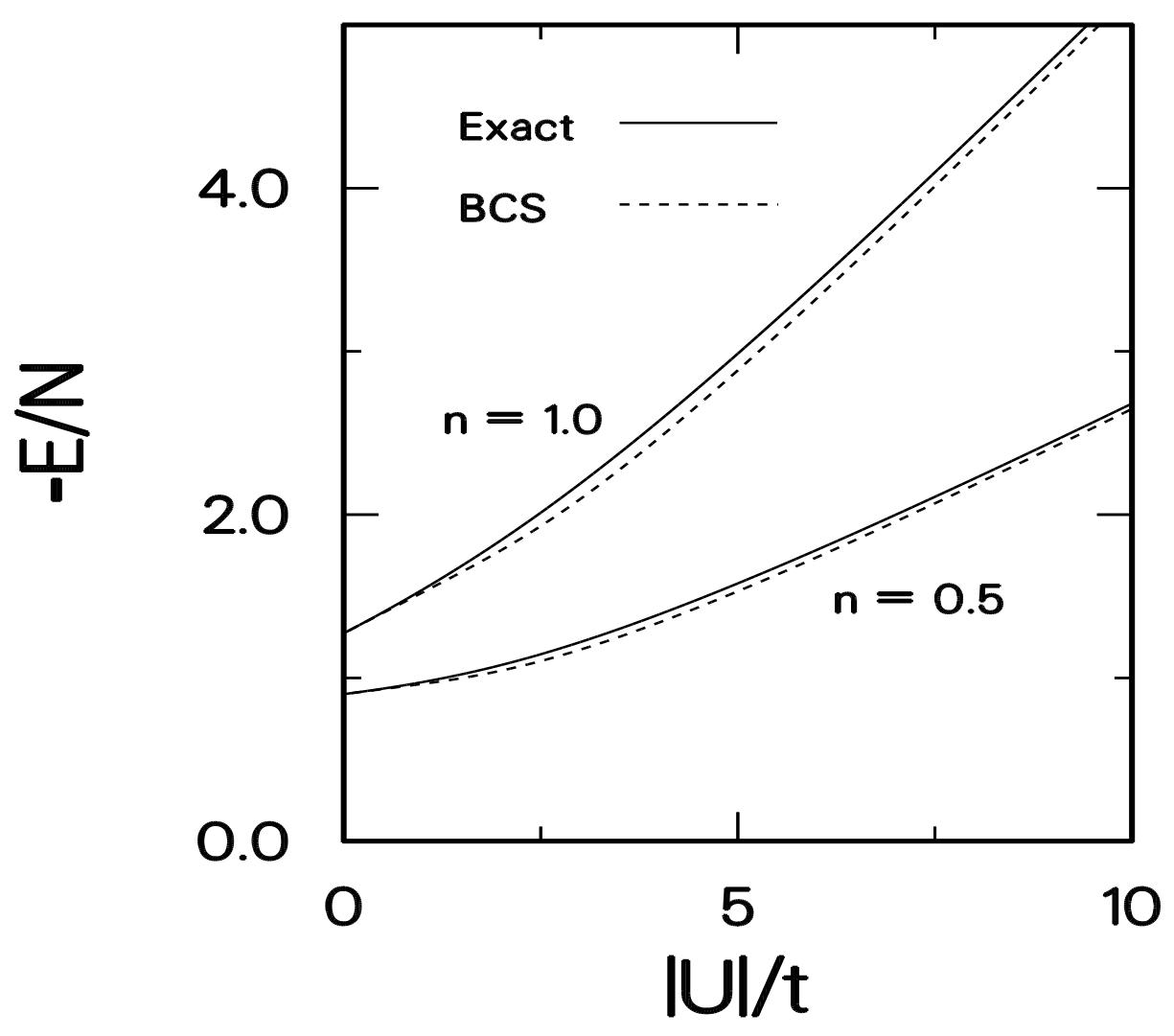

FIG. 2. The ground state energy as a function of the coupling strength for quarter $(n=0.5)$ and half $(n=1.0)$ filling, along with the BCS results. 
Fig. 3a Marsiglio

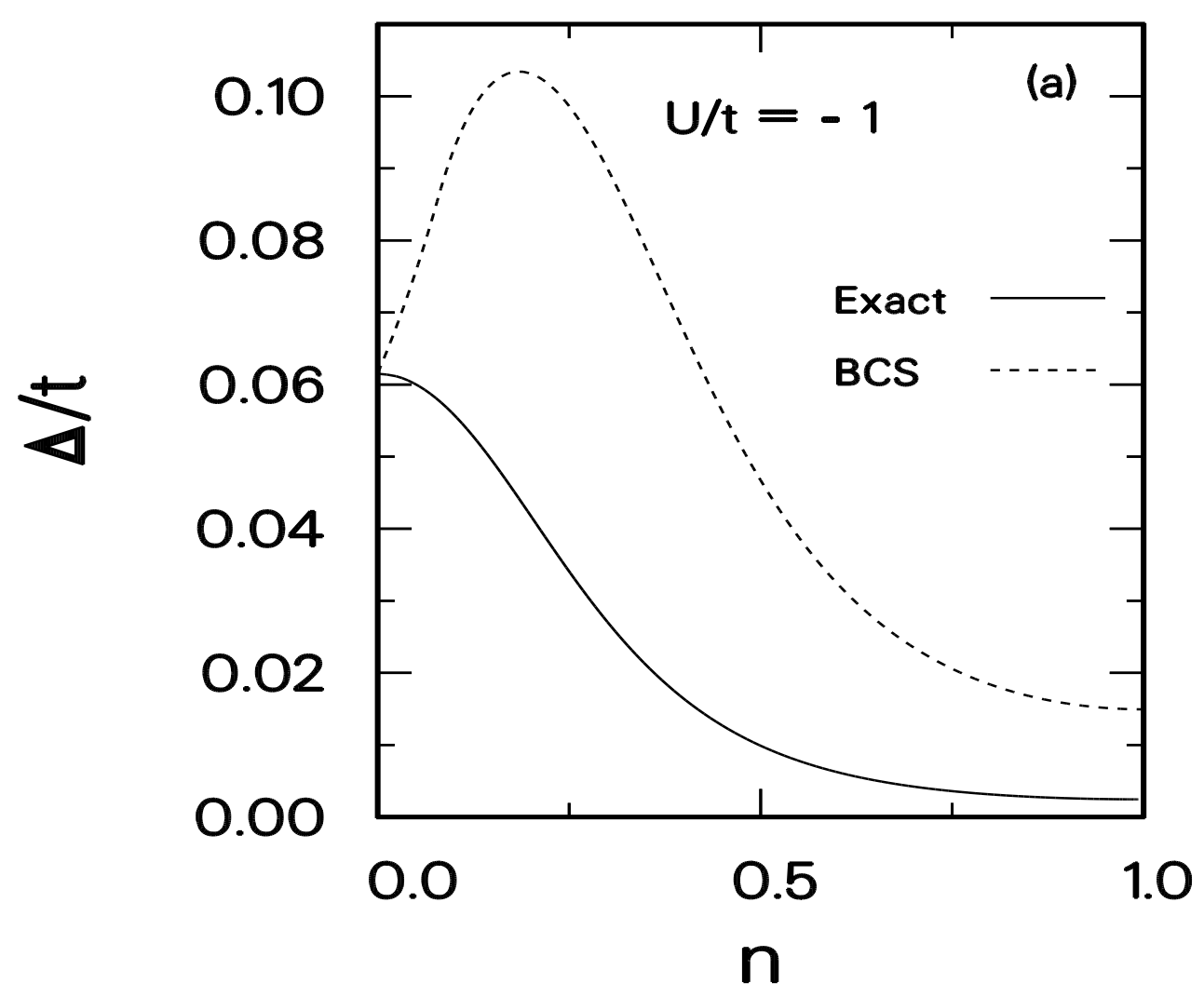

FIG. 3. The energy gap, $\Delta / t$ vs. electron density for (a) $|U| / t=1$, (b) $|U| / t=2$, and (c) $|U| / t=10$. The BCS result (i.e. $\Delta_{\circ} / t$ ) is shown for comparison. 
Fig. 3b Marsiglio

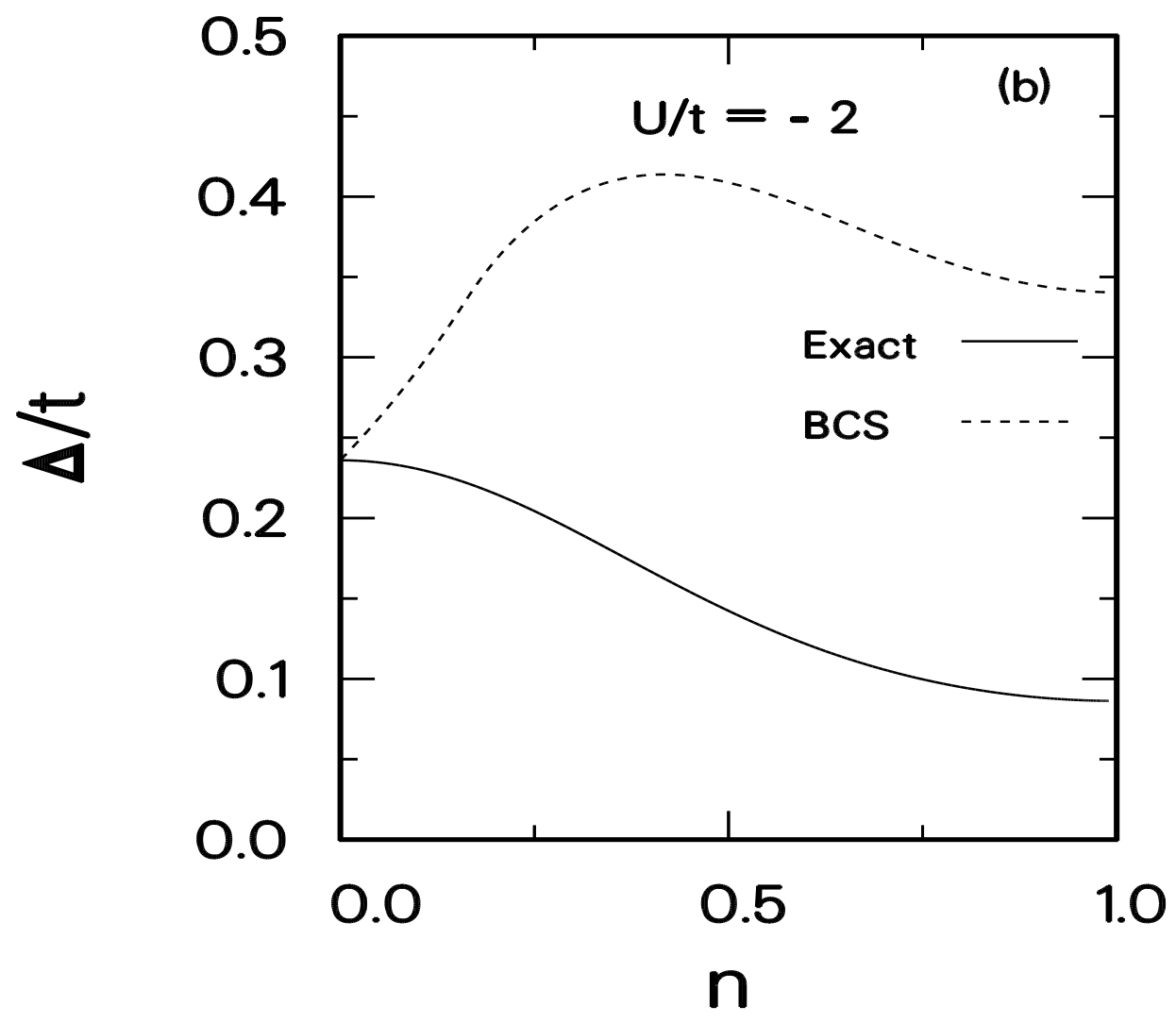


Fig. 3c Marsiglio

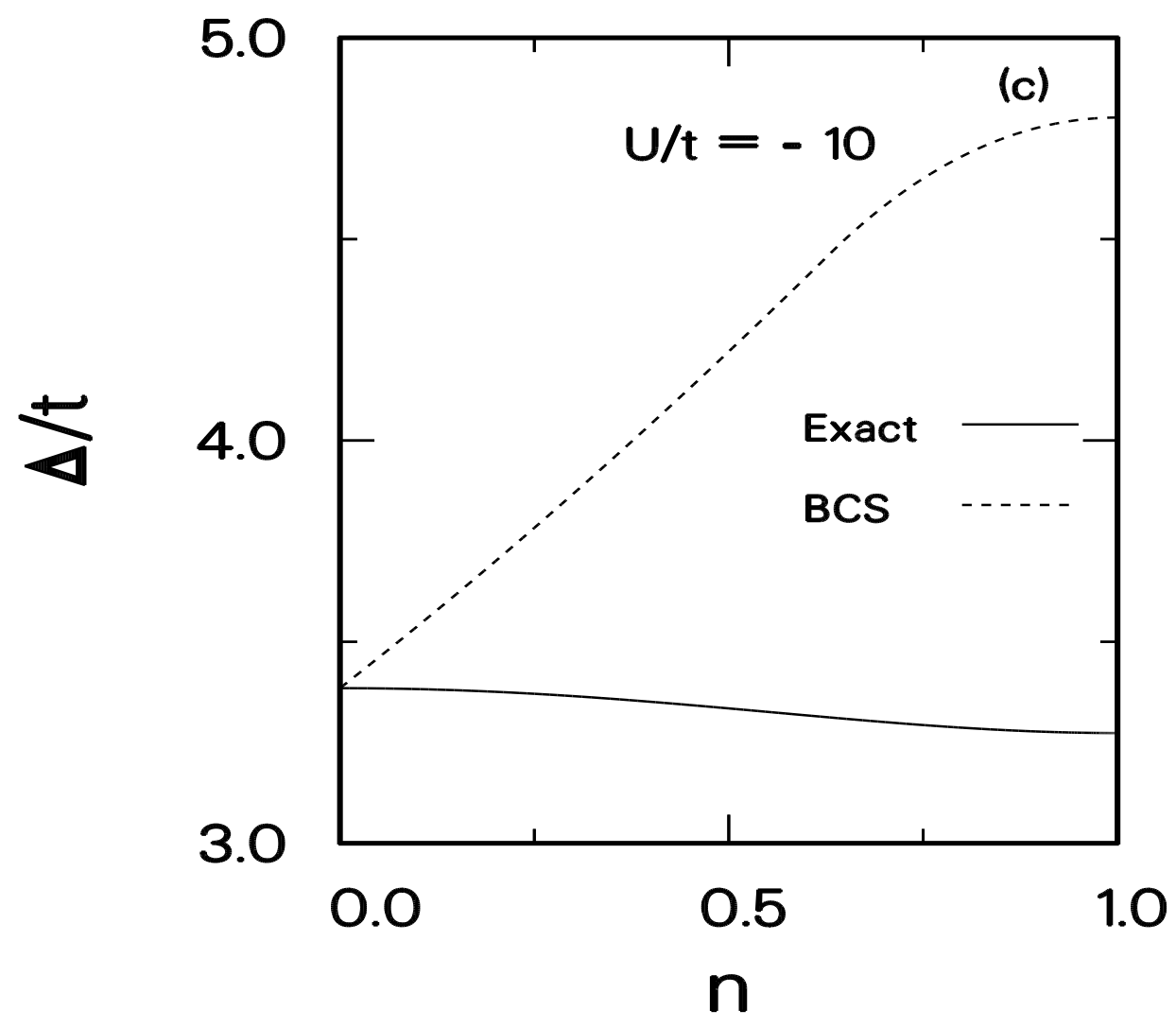


Fig. 4a Marsiglio

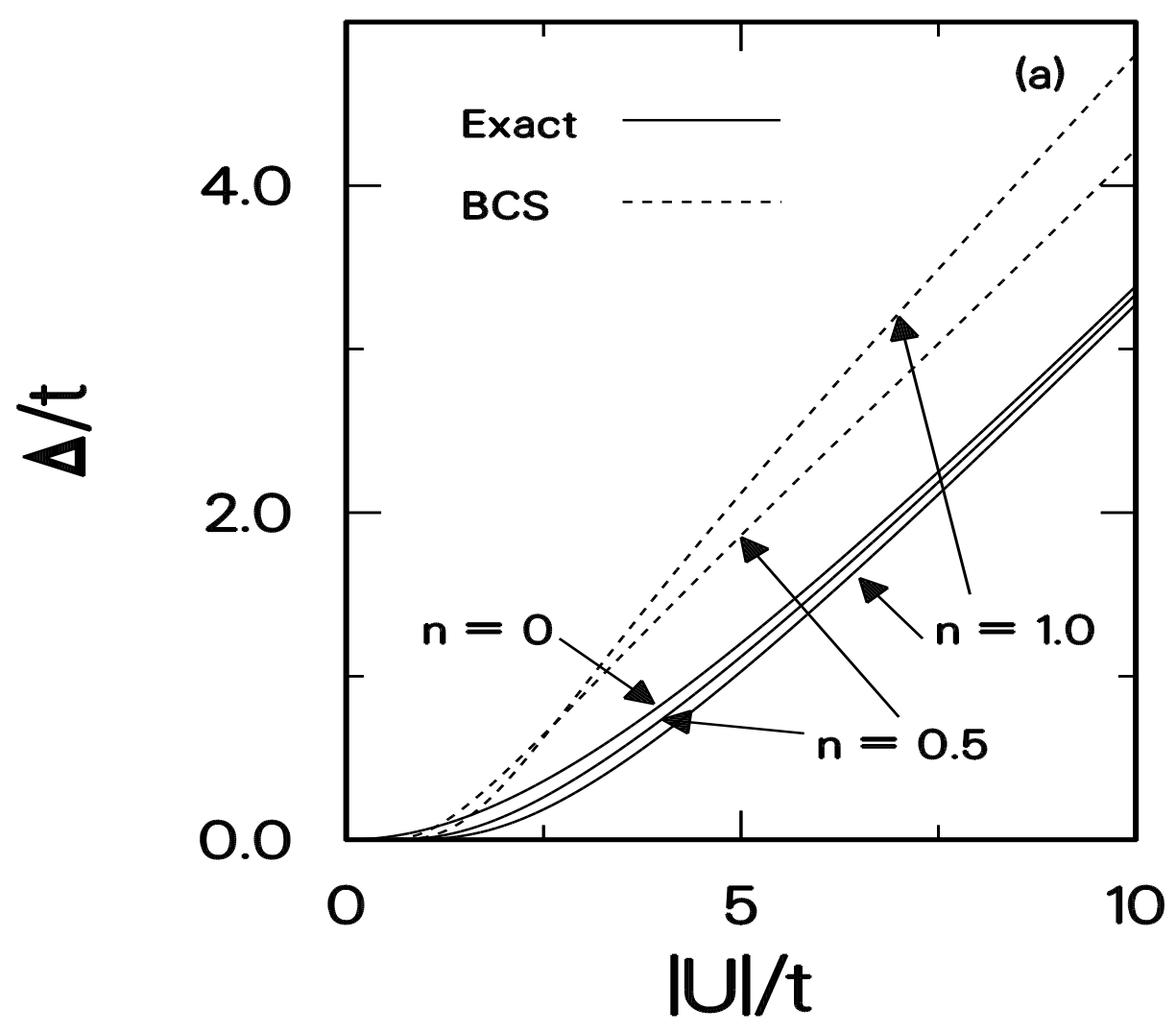

FIG. 4. (a) The energy gap, $\Delta / t$ vs. coupling strength, $|U| / t$, for various electron densities. In (b) we provide an expanded version of the weak coupling regime. 
Fig. 4b Marsiglio

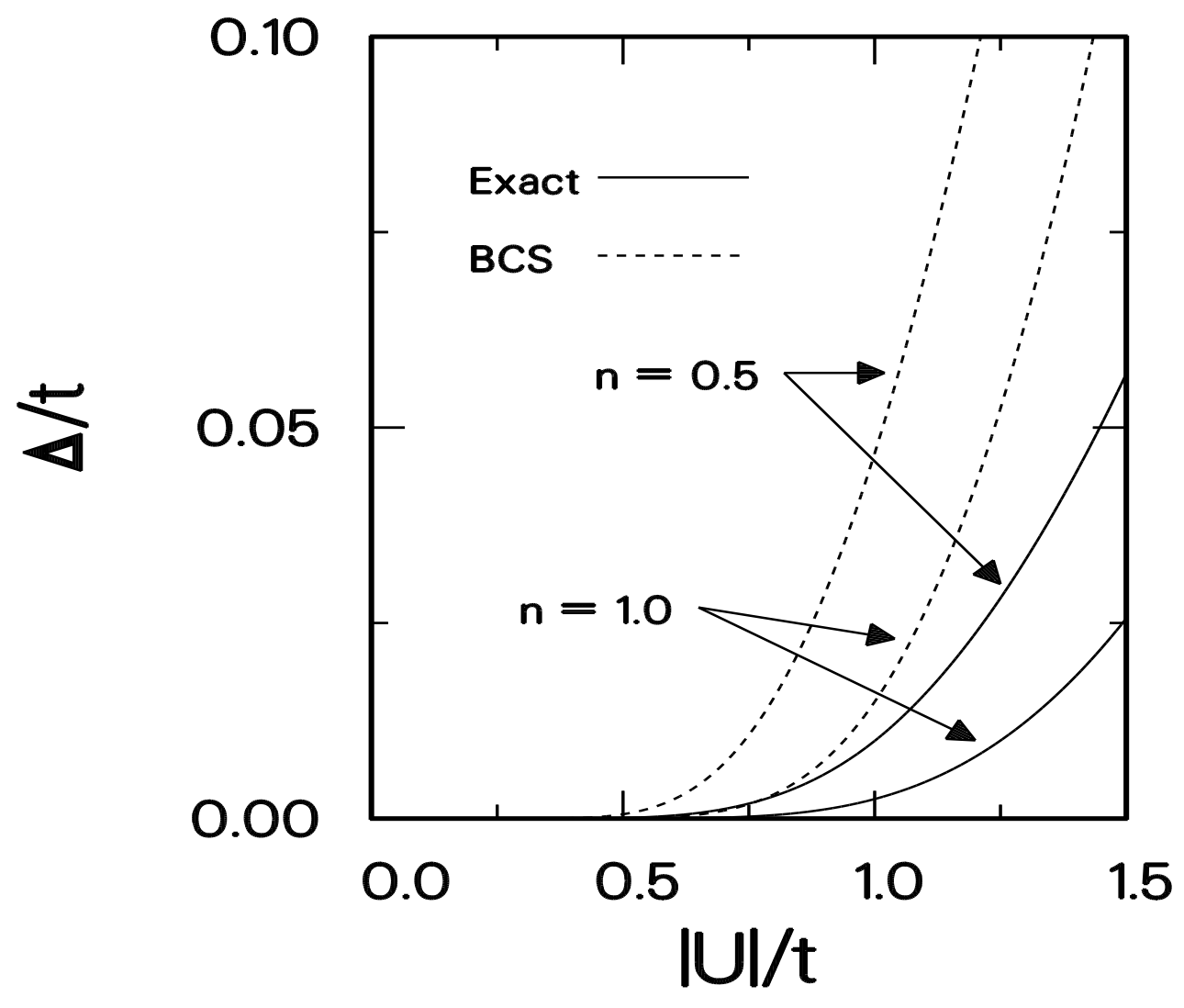


Fig. 5 Marsiglio

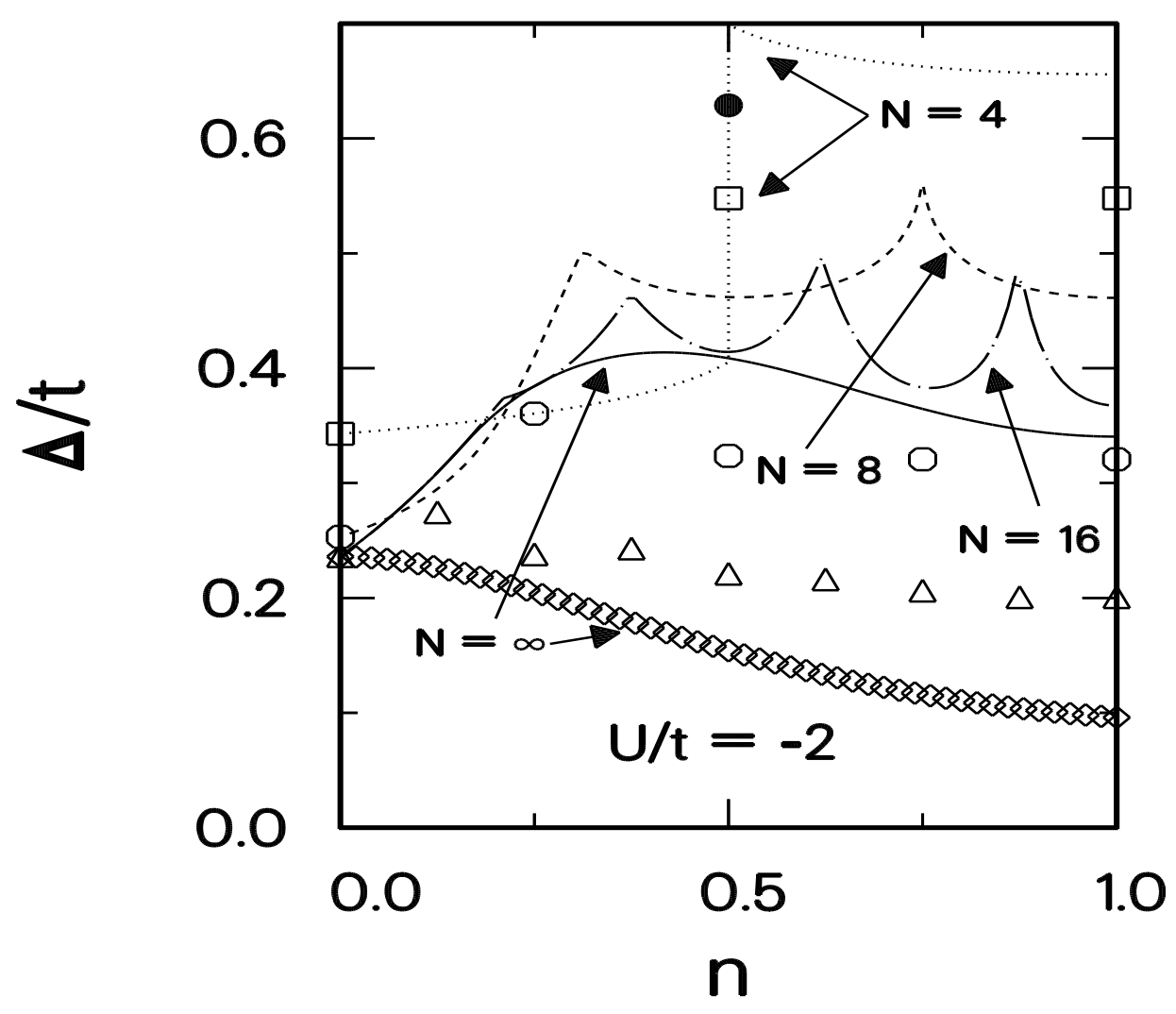

FIG. 5. The energy gap, $\Delta / t$ vs. electron density for $|U| / t=2$, for various chain sizes. The open symbols are from exact results, with lattice size as indicated. The curves are BCS results (using the grand canonical ensemble) with lattice sizes as indicated. We have also indicated the result for quarter-filling for $N=4$ (filled circle), showing an improvement over the grand canonical result. 